\title{
DENGUE, MOVILIDAD TERRITORIAL Y RELATO EPIDEMIOLÓGICO: DIAGNÓSTICOS Y EXPLICACIONES ESTATALES SOBRE LAS EPIDEMIAS DE 2009-2010 Y 2015-2016 EN ARGENTINA
}

\section{Dengue, territorial mobility and epidemiological report: diagnoses and state explana- tions about the epidemics of 2009-2010 and 2015-2016 in Argentina}

\section{Laura Gottero*}

Recibido: 15/12/2017 Aprobado: 14/4/2018

Cómo citar: Gottero L, Dengue, movilidad territorial y relato epidemiológico: diagnósticos y explicaciones estatales sobre las epidemias de 2009-2010 y 2015-2016 en Argentina Ciencia y Salud [Internet]. 2018 mayo 1; [citado 2018 agosto 15]; 2(2):[aprox. 0 p.]. Disponible en: https://revistas.intec.edu.do/index.php/cisa/article/view/1178

\section{Resumen}

Este artículo realiza un análisis comparativo de la producción de información pública sobre las dos últimas epidemias de dengue sucedidas en Argentina, en los años 2009 y 2016 respectivamente. Mediante un análisis de fuentes documentales, se realiza un recorrido crítico de la construcción del relato epidemiológico estatal sobre esta enfermedad en ambos lapsos, donde se observa la preeminencia del argumento de la movilidad y de los antecedentes de viaje como casi única explicación para los brotes, en desmedro de un estudio contextuado de la problemática que indague sobre determinantes sociales y modelos de explotación económicos que inciden fuertemente en el ambiente.

Palabras clave: dengue; epidemias; información pública; usos de la epidemiología, migración.

\footnotetext{
* Instituto de Justicia y Derechos Humanos, Universidad Nacional de Lanús, Argentina. lauritagottero@gmail.com
}

\begin{abstract}
This article makes a comparative analysis of the production of public information on the last two dengue epidemics occurred in Argentina, in 2009 and 2016 respectively. Through an analysis of documentary sources, a critical path of the construction of state epidemiological account of this disease in both periods is made, where is analized the preeminence of argument mobility and travel background like almost only explanation for outbreaks observed at the expense of a contextualized study of the problems inquire about social determinants and models of economic exploitation that strongly influence the environment.
\end{abstract}

Keywords: dengue epidemics; public information; uses of epidemiology; migration. 


\section{Introducción}

En este artículo se discutirá la preponderancia del argumento de la movilidad de personas en los diagnósticos oficiales que construyen las explicaciones acerca de las epidemias de dengue. Si bien se considerará que, desde un planteo epidemiológico que solo observa la posibilidad de contagio, estos factores puedan tener importancia, su referencia casi exclusiva en reemplazo de otros factores o dimensiones impide la construcción de respuestas renovadas cuando las razones migratorias o fronterizas parecen haber agotado su capacidad heurística -o, en muchos casos, no han contado siquiera con esta condición-.

\section{Marco teórico}

Un grupo creciente de producciones científicas sobre enfermedades transmisibles ${ }^{1-6} \mathrm{y}$ documentos sanitarios estatales e internacionales ${ }^{7,8}$ tiende a enumerar el factor de la migración y/o de la movilidad de personas como un elemento de importancia entre las razones para la expansión de una enfermedad, pero son menos los estudios que discuten sobre esas afirmaciones desde estos mismos enfoques. Si bien se observan investigaciones que realizan aportes sustanciosos sobre la articulación entre migración y salud ${ }^{9-12}$, suelen anclarse en perspectivas de estudios migratorios y/o de abordajes interculturales que no llegan a discutir esos diagnósticos construidos y no ponen en cuestión los discursos sobre la movilidad de personas asociados con la situación de salud de un territorio ${ }^{13-15}$.

La discusión que propone este artículo parte del seguimiento pormenorizado de los informes periódicos que el Estado argentino elaboró durante los seis meses que, en promedio, duró cada epidemia en los dos lapsos señalados (2009-2010 y 2015-2016); en la primera etapa, los Partes de Situación sobre el Dengue; en la segunda, los Boletines Integrados de Vigilancia (sección Dengue). La investigación se realizó de acuerdo con un diseño cualitativo que combinó herramientas metodológicas para adecuar teorías y métodos al objeto de estudio para desarrollar un diseño flexible y emergente ${ }^{16-18}$. Se efectuó una lectura crítica de los informes y la construcción de los cuadros que sintetizan las observaciones -presentados en este artículo-, de acuerdo con un enfoque de análisis del discurso ${ }^{19-23}$, que privilegia el estudio de las vinculaciones entre lo ideológico y lo lingüístico, y considera un enfoque interdisciplinario para la elaboración de conclusiones sustentado en la idea de transdepartamentalidad elaborada por Matus ${ }^{25}$.

\section{Resultados}

\section{Informes de situación: análisis comparativo entre los dos eventos epidémicos}

Los Partes de Situación y los Boletines Integrados de Vigilancia son los informes técnicos elaborados por el Ministerio de Salud argentino para equipos de salud y responsables políticos que, durante las dos epidemias, configuraron el diagnóstico estatal sobre el dengue en Argentina ${ }^{26-27}$. Para el seguimiento realizado, se seleccionaron los Partes publicados entre noviembre de 2009 y junio de 2010, y se relevaron los Boletines entre septiembre de 2015 y abril de 2016, desde la aparición de los primeros casos hasta el descenso en la curva de notificaciones.

Los datos obtenidos se distribuyeron en dos ejes de estudio: a) la situación nacional en cuanto al conteo y la localización de casos; b) las referencias o menciones a la movilidad de personas o la frontera como factores causales, así como la relación numérica entre casos con antecedentes de viaje $(\mathrm{AV})$ y sin antecedentes de viaje (sin AV) en cada informea.

\section{Partes de Situación de Dengue en Argentina (2009-2010)}

A continuación, se presenta una síntesis y selección de algunos elementos relevantes identificados 
en estos documentos, recortados desde el inicio de la circulación viral. La discusión y las conclusiones abrevan, sin embargo, en el seguimiento completo de estos partes, que no se incluyen aquí por razones de espacio.

Desde el inicio de los informes, la distribución inicial de notificaciones de dengue no se sitúa, en su mayoría, en la frontera o en las provincias fronterizas, sino en el sur de Santa Fe y Salta. Así, el inicio de la epidemia no está marcado por la movilidad internacional ni por los $\mathrm{AV}$ internos; no obstante, siempre se aclara el AV en notificaciones de provincias fronterizas y no fronterizas.

La construcción simbólica de la frontera noreste-noroeste como territorio sujeto a múltiples sentidos ha sido analizada en numerosos trabajos, de los cuales los relacionados con la salud han sido los menos frecuentes ${ }^{28-31}$. No obstante, la consideración de los territorios fronterizos como susceptibles de mayor atención sanitaria constituye una constante discursiva en el discurso estatal, de allí su relevancia para el estudio. Esto se observa en la paridad de notificaciones en Santa Fe, Misiones y Salta (parte 12), por lo que el "factor frontera" no parece jugar un rol preponderante allí. Además, se observa el inicio de un brote en Chaco (Corzuela) sin AV y, por lo tanto, no relacionado con la movilidad.

Desde la geografía de la salud hay trabajos que focalizan en la parcialidad de los registros que impiden la construcción de explicaciones complejas para problemáticas sanitarias ${ }^{32-34}$, lo que permite

\section{Cuadro No. 1. Partes de situación del dengue en Argentina}

\begin{tabular}{|c|c|c|}
\hline Parte de Situación & Conteo/localización - Situación nacional & Movilidad de personas - Antecedentes de viaje \\
\hline $12(19 / 2 / 2010)$ & $\begin{array}{l}\text { Circulación viral en todo el país. Misiones, Chaco, } \\
\text { Santa Fe, CABA y provincia de Buenos Aires con } \\
\text { casos confirmados. } \\
18 \text { provincias con notificaciones en aumento: } \\
\text { Misiones ( } 221) \text {, provincia de Buenos Aires (126), } \\
\text { Santa Fe (110) y Salta (95), entre otras. }\end{array}$ & $\begin{array}{l}\text { Los } 23 \text { casos en Corzuela no tienen AV. Un caso en } \\
\text { La Plata (Bs. As.) con AV interno (Puerto Iguazú). }\end{array}$ \\
\hline $13(26 / 2 / 2010)$ & $\begin{array}{l}\text { Desde inicio de año, } 544 \text { casos confirmados de } \\
\text { dengue en Misiones (495), Chaco (34), Buenos } \\
\text { Aires (189) y Chaco (121). }\end{array}$ & $\begin{array}{l}\text { En Chaco, } 2 \text { de } 34 \text { casos tienen AV a Misiones. En } \\
\text { Buenos Aires, un caso con AV "a zonas afectadas". } \\
\text { En Santa Fe, } 1 \text { de } 3 \text { casos tiene AV a Brasil. }\end{array}$ \\
\hline $16(19 / 3 / 2010)$ & $\begin{array}{l}656 \text { casos desde el inicio de } 2010 \text {, localizados en } \\
\text { Misiones (535), Chaco ( } 75) \text {, Entre Ríos (3), Salta } \\
\text { (2), Buenos Aires (2) y Santa Fe (3). } \\
\text { La mayor cantidad de notificaciones se dio en } \\
\text { Santa Fe (31), seguida por Chaco (29). }\end{array}$ & $\begin{array}{l}\text { Buenos Aires: } 3 \text { de } 4 \text { casos tienen AV. } \\
\text { Chaco: de los } 97 \text { casos, hay } 4 \text { con AV (Misiones y } \\
\text { Corzuela). } \\
\text { Entre Ríos: } 3 \text { casos con AV (Bolivia). Salta: de } 47 \\
\text { casos, } 3 \text { han confirmado AV. Santa Fe: de } 26 \text { casos, } \\
1 \text { tiene AV. }\end{array}$ \\
\hline $\begin{array}{l}23(4 / 6 / 2010) \text {, con actualización de } \\
\text { datos al 30/5. }\end{array}$ & $\begin{array}{l}1185 \text { pacientes con dengue desde el } 1^{\circ} \text { de enero. } \\
\text { Santiago del Estero: hay un brote de dengue en } \\
\text { Clodomira, con } 78 \text { enfermos. } 8 \text { casos sin AV en la } \\
\text { ciudad capital y en Ańatuya. } \\
\text { Situación por provincias: CABA, 5; Buenos } \\
\text { Aires, 25; Catamarca, 1; Chaco, 93; Chubut, 1; } \\
\text { Corrientes, 11; Entre Ríos, 3; Formosa, 1; Jujuy, } \\
\text { 6; La Pampa, 2; Mendoza, 1; Misiones, 872; Salta, } \\
\text { 62; Santa Fe, 32; Santiago del Estero, 78. }\end{array}$ & $\begin{array}{l}\text { Buenos Aires: de } 25 \text { casos, hay } 3 \text { con AV. } \\
\text { Jujuy: } 6 \text { casos con AV. } \\
\text { La Pampa: } 2 \text { casos con AV. } \\
\text { Mendoza: } 1 \text { caso con AV (Bolivia). } \\
\text { Salta: brote en Orán de } 59 \text { casos sin AV; } 3 \text { casos en } \\
\text { otras localidades con AV (Bolivia). } \\
\text { Santa Fe: de } 32 \text { casos, hay } 1 \text { con AV. }\end{array}$ \\
\hline
\end{tabular}

Fuente: elaboración propia a partir del análisis de los Partes de Situación publicados por el Ministerio de Salud de la Nación (Argentina). 
preguntarse por qué la atención se focaliza en la constatación de AV mientras las notificaciones (sin AV) siguen aumentando en Santa Fe. Lo anterior se evidencia en el parte 16, que destaca un AV de migración interna a Chaco -lo que replica la lógica causal de la movilidad internacional sobre las trayectorias al interior del territorio argentino-, y en la preeminencia a los datos de AV cuando los hay, a pesar de que forman una minoría respecto del total. El desplazamiento internacional y nacional de personas constituye casi la única dimensión que los registros se proponen rastrear, a pesar de que no sea fructífero a la hora de evaluar, por ejemplo, el brote autóctono en Chaco o los casos sin AV en Santa Fe.

El último parte publicado durante la epidemia es el 23, con datos actualizados hasta el 30/5. En una fase de multiplicación de los brotes, la suspensión de los informes impide su seguimiento, pero los datos obtenidos permiten destacar que: i) se observa un énfasis en la constatación de AV de los casos confirmados; ii) no existen explicaciones complementarias para las provincias no fronterizas donde se registran brotes y casos muy numerosos sin AV. Así, el registro de casos destaca los grupos de notificaciones que parecen explicarse solo por el argumento de la movilidad, mientras que los que no están asociados con los viajes carecen de explicaciones causales sobre la epidemia.

\section{Boletines Integrados de Vigilancia Epidemiológica (2015-2016)}

Los Boletines Integrados de Vigilancia Epidemiológica muestran un cambio en la producción de información pública sobre dengue desde el Estado. Si bien los reportes sobre esta enfermedad no se publican en un documento aparte y se integran al panorama epidemiológico nacional, se observan cambios de las explicaciones con respecto al periodo anterior. Surgen nuevos datos a partir de la diversificación de categorías y de la especificación territorial en el registro, lo que modifica en parte el relato estatal. El modo de recorte y síntesis de datos fue el mismo que el de los Partes, por lo que se presentan aquí algunos elementos significativos para el análisis.

El Boletín 275 muestra que el primer brote de la epidemia se registra en una provincia no fronteriza (Córdoba), donde la cantidad de casos autóctonos supera desde el inicio a los “importados". El registro de los AV no permite brindar explicaciones alternativas para este brote, que en su abrumadora mayoría presenta casos sin AV. El ejemplo de esta provincia funciona de manera similar con la importancia de Santa Fe en la epidemia anterior, como provincia con casos notificados y confirmados sin AV sobre la que no se elaboran explicaciones alternativas al argumento de la movilidad territorial.

Hasta esta instancia de publicaciones, la categoría de "importados" indica que no se produjeron en el lugar relevado, pero no distingue entre movilidad interna e internacional, y oculta las condiciones de producción del vector en localidades argentinas con elevada desigualdad estructural. En consecuencia, no permite profundizar la indagación respecto de las transformaciones en el patrón de expansión y circulación del dengue reconocidas por la misma publicación $^{1 \mathrm{~b}}$.

El Boletín 289 muestra que hay una lenta confirmación de casos autóctonos y mayor velocidad en el registro y la difusión de casos importados (movilidad interna con la internacional), lo que contribuye con la argumentación estatal. A partir de esta instancia del ciclo epidémico, los datos muestran que el mayor número de notificaciones (y de casos

1b. "El período epidémico de 2015 en Argentina se caracterizó por su inicio en la ciudad de Córdoba, seguido una concentración de casos sin registro de viaje en Buenos Aires y CABA. Este patrón se diferencia del observado en años anteriores en los que las localidades inicialmente afectadas fueron las limítrofes con países endémicos, para luego extenderse a otras más alejadas" (Boletín 287). Pese a este reconocimiento, no se incluyen explicaciones alternativas a la de la movilidad para comprender este nuevo escenario. 


\section{Cuadro No. 2. Boletines Integrados de Vigilancia Epidemiológica - Sección Dengue}

\begin{tabular}{|c|c|c|}
\hline Boletín & Situación nacional - Conteo y localización & $\begin{array}{l}\text { Movilidad - Antecedentes de viaje nacional o } \\
\text { internacional }\end{array}$ \\
\hline № 275 - SE 36 - septiembre 2015 & $\begin{array}{l}\text { Semana 1-35: } 257 \text { casos. } \\
\text { Mayor número de notificaciones: Córdoba (36\%) } \\
\text { y Salta }(12 \%) \text {. }\end{array}$ & $\begin{array}{l}\text { De los } 257 \text { casos confirmados, } 182 \text { son autóctonos } \\
\text { (A) y } 75 \text { "importados" (I). Más del } 90 \% \text { de casos } \\
\text { autóctonos se localiza en Córdoba capital (lugar del } \\
\text { primer brote). }\end{array}$ \\
\hline No 282 - SE 43 - octubre 2015 & $\begin{array}{l}344 \text { casos confirmados: } 265 \text { autóctonos y } 79 \text { "con } \\
\text { AV". }\end{array}$ & $\begin{array}{l}\text { Bs. As.: } 11 \text { A. y } 15 \text { I; CABA: } 19 \text { A y } 28 \text { I; Córdoba: } \\
235 \text { A y } 11 \text { I; Sta. Fe: } 0 \text { A, } 1 \text { P y } 13 \text { I. }\end{array}$ \\
\hline No 289 - SE 50 - diciembre 2015 & $\begin{array}{l}349 \text { casos confirmados: } 265 \text { A y } 84 \text { con AV interno } \\
\text { e internacional. }\end{array}$ & $\begin{array}{l}6 \text { nuevos casos: } 1 \text { en Tucumán, } 1 \text { en Buenos Aires } \\
\text { y } 1 \text { en Córdoba con AV a Paraguay; } 1 \text { en Córdoba } \\
\text { y } 1 \text { en Jujuy con AV a Clorinda; } 1 \text { en Santa Fe con } \\
\text { AV a Misiones. }\end{array}$ \\
\hline No 298 - SE 8 - febrero 2016 & $\begin{array}{l}6389 \text { casos confirmados: } 5083 \text { autóctonos, } \\
\text { distribuidos en } 14 \text { jurisdicciones del país. }\end{array}$ & $\begin{array}{l}\text { Del total de casos } 1226 \text { tienen AV a } 21 \text { provincias. } \\
\text { De estos, } 479 \text { tienen AV a Formosa o Misiones, y } \\
\text { el resto AV internacional (especialmente Paraguay } \\
\text { y Brasil). }\end{array}$ \\
\hline № 305 - SE 15 - marzo 2016 & $\begin{array}{l}\text { Brotes de dengue con transmisión sostenida en: } \\
\text { Buenos Aires, CABA, Córdoba, Corrientes, Chaco, } \\
\text { Entre Ríos, Formosa, Jujuy, Misiones, Salta y Santa } \\
\text { Fe. } \\
\text { Transmisión circunscripta a localidades, barrios o } \\
\text { conglomerados con casos autóctonos y probables } \\
\text { en: Catamarca, Santiago del Estero y Tucumán. }\end{array}$ & $\begin{array}{l}\text { De los } 2735 \text { casos confirmados o probables, } \\
24.879 \text { son autóctonos y están distribuidos en } 15 \\
\text { jurisdicciones del país, y } 2356 \text { son importantes, } \\
\text { distribuidos en } 23 \text { provincias. } \\
\text { El mapa de casos autóctonos muestra su } \\
\text { localización desde el centro hacia el norte y este } \\
\text { del país. Hay zonas de frontera en el NOA que } \\
\text { no estarían afectadas (como toda la frontera de la } \\
\text { provincia Jujuy). }\end{array}$ \\
\hline
\end{tabular}

Fuente: elaboración propia a partir del análisis de los Boletines Integrados de Vigilancia publicados por el Ministerio de Salud de la Nación (Argentina).

autóctonos) sigue estando en Córdoba, y que los casos sin $\mathrm{AV}$ se encuentran tanto en provincias de frontera como en aquellas que no lo son ${ }^{2 c}$ (Boletines 290 y 293 no incluidos en el cuadro).

En los posibles casos autóctonos sin $\mathrm{AV}$ evidentes, se rastrea el esquema familiar hasta encontrar personas de su entorno que han viajado ${ }^{3 c}$, sin aportar datos

2c. A pesar de las conclusiones que surgen de estas comparaciones, el hincapié puesto en la movilidad permite afirmar que "El riesgo de dispersión de la enfermedad a otras jurisdicciones es alto, dado que se registran casos importados (n. de la a.: movilidad interna) en la mayoría de las provincias con vector" (Boletín 292) y, a la vez, que por motivo de las vacaciones de verano y la estimación del aumento del flujo de viajeros internos e internacionales aumentará, por lo que hay que tomar medidas extra para contrarrestar el riesgo de propagación del virus.

3c Hay un caso en Santa Fe que corresponde a una persona que no viajó, pero "como producto de la investigación pudo de indagaciones socioambientales o socioeconómicas que puedan ofrecer otros enfoques a la problemática. El Boletín 298 muestra cuantitativamente las insuficiencias del argumento de la movilidad: mientras el $20 \%$ de los casos confirmados son "importados" y pueden inscribirse en este razonamiento -si bien una tercera parte está relacionada con el AV interno-, el $80 \%$ restante no puede explicarse mediante la existencia de AV, pero para esta mayoría no hay causas alternativas expresas. Finalmente, el Boletín 305 indica una disminución de las notificaciones que anticipa el descenso en la curva epidémica. Si bien la estadística enfoca con mayor interés la distribución de casos autóctonos y en su progresión respecto del 2009, no se elaboran hipótesis ni

establecerse el nexo con viajeros a las provincias de Formosa y Paraguay” (Boletín 294, pp.13). 
Cuadro No. 3. Hogares con tipo de provisión de agua, por provincia (Argentina, 2010)

\begin{tabular}{lrrrr}
\hline Provincia & $\begin{array}{l}\text { Cantidad de } \\
\text { hogares }\end{array}$ & $\begin{array}{l}\text { Provición de agua } \\
\text { dentro de la vivienda }\end{array}$ & $\begin{array}{l}\text { Provisión de agua fuera de la } \\
\text { vivienda y dentro del terreno }\end{array}$ & $\begin{array}{l}\text { Provisión de agua fuera de la vi- } \\
\text { vienda y dentro del terreno }\end{array}$ \\
\hline Jujuy & 174.630 & $142.373(81,53 \%)$ & $22.610(12,95 \%)$ & $9.647(5,52 \%)$ \\
Salta & 299.794 & $229.157(76,44 \%)$ & $53.198(17,74 \%)$ & $17.439(5,82 \%)$ \\
Santiago del Estero & 218.025 & $128.078(58,74 \%)$ & $167.366(19,3 \%)$ & $167.366(19,3 \%)$ \\
Formosa & 140.303 & $82.682(58,74 \%)$ & $40.169(28,63 \%)$ & $17.452(12,44 \%)$ \\
Chaco & 288.422 & $187.688(65,07 \%)$ & $66.333(23 \%)$ & $34.401(11,93 \%)$ \\
Misiones & 302.953 & $225.121(74,31 \%)$ & $57.868(19,10 \%)$ & $19.964(6,59 \%)$ \\
\hline
\end{tabular}

Fuente: INDEC, Resultados definitivos Censo 2010. Cuestionario básico. Base de datos REDATAM.

conclusiones respecto de este aumento, aunque la serie finaliza con porcentajes muy superiores para los casos autóctonos respecto de los importados, además de observarse un crecimiento más veloz de aquellos desde los inicios de la epidemia. Además, hay zonas de frontera que no se encuentran afectadas (Jujuy), mientras que hay zonas del centro que sí lo están (Córdoba, Buenos Aires, Santiago del Estero).

\section{Discusión de los resultados}

\section{Caracterización territorial de los brotes: explicaciones alternativas}

Las estadísticas producidas por el Estado permiten elaborar otro tipo de explicaciones acerca de las epidemias de dengue en Argentina. Aquí se presentan dos de estos argumentos no dominantes que surgen de la discusión actual sobre la prevalencia de dengue en América Latina ${ }^{4 \mathrm{~d}}$, para los cuales bastan los datos oficiales.

a. Desigualdades socioeconómicas estructurales

Las corrientes epidemiológicas alternativas establecen la variable de la desigualdad como dimensión

\footnotetext{
4d. Médicos de Pueblos Fumigados (Argentina) y ABRASCO (Brasil), por ejemplo, se manifestaron enérgicamente en pos de reconocer la importancia de los determinantes sociales de la salud en las epidemias de dengue y zika.
}

explicativa insoslayable de los eventos de saludenfermedad, tanto desde la medicina social como desde la salud colectiva ${ }^{35-41}$.

Un análisis comparativo entre las provincias argentinas más afectadas por los brotes de dengue en el 2009 y en 2015 -repetidas en ambos eventos- y los datos sobre tenencia de agua disponibles en el Censo Nacional 2010 (INDEC, 2010) ${ }^{42}$, posibilitan resaltar una relación entre la incidencia de dengue y las deficiencias en la infraestructura que restringen el derecho al agua potable en diversas zonas de Argentina.

Las provincias donde surgieron brotes intensos de dengue son también aquellas donde se constatan porcentajes considerables de hogares sin provisión de agua dentro de la vivienda. En el caso de Santiago del Estero, Chaco y Formosa, también existen índices considerables de hogares que no tienen agua potable dentro de su terreno y que deben recurrir a fuentes disponibles en las cercanías. Esta situación constituye la principal causa de las prácticas señaladas como altamente riesgosas para la difusión del dengue: la acumulación de agua limpia en recipientes por falta de disponibilidad constante. Esta acción obligada para los habitantes de zonas vulnerables de las provincias señaladas promueve la proliferación del mosquito ${ }^{43-44}$.

En todas estas provincias afectadas por el dengue -tanto las fronterizas como las que no se 


\section{Cuadro No. 4. Superficie implantada y producción de soja en Argentina. Período 2009-2014}

\begin{tabular}{lcc}
\hline & $\begin{array}{l}\text { Superficie implantada de soja y superficie total im- } \\
\text { plantada de oleaginosas (miles de ha) }\end{array}$ & $\begin{array}{l}\text { Producción de soja y producción total de oleaginosas } \\
\text { (miles de tn) }\end{array}$ \\
\hline $2009-2010$ & $18.343,3$ & $52.676,6$ \\
$2010-2011$ & $18.886,6$ & $48.885,7$ \\
$2011-2012$ & $18.670,9$ & $40.100,2$ \\
$2012-2013$ & $20.035,6$ & $49.306,2$ \\
$2013-2014$ & $19.781,8$ & $53.397,7$ \\
\hline
\end{tabular}

Fuente: elaboración propia sobre la información del Ministerio de Agricultura, Ganadería y Pesca, Dirección de Información Agropecuaria y Forestal (Argentina).

encontraban cerca de países limítrofes-, la movilidad fue casi la única explicación acerca de los brotes sucedidos en su territorio. Sin embargo, el análisis de estadísticas sobre acceso a agua potable permite visibilizar cómo, antes que provincias de frontera, estas son regiones afectadas por la desigualdad, en términos de salud y por la persistencia de factores estructurales que aumentan el riesgo de dengue.

B. Modelo productivo de la soja: impacto ambiental y sanitario

En los últimos años, la discusión sobre modelos productivos y acumulación del capital en Sudamérica se vio complementada por una línea de investigación compuesta por estudios de las ciencias de la salud, ambientales y económicos que destacan la incidencia ambiental y sanitaria del cultivo extensivo de soja en los países del Sur de América, como Paraguay, Brasil y Argentina ${ }^{45-47}$.

En ese contexto, una articulación de fuentes estadísticas oficiales, combinadas con los "mapas del dengue" que forman parte de la información epidemiológica estatal, permiten observar vinculaciones significativas ${ }^{48}$ con este tipo de producción que deberían ser tenidas en cuenta por una estrategia sanitaria de prevención y control.

En el conjunto de las oleaginosas más importantes la soja consolidó desde hace casi diez años su preeminencia en el conjunto de cultivos de este tipo, y representa el 55\% del total de hectáreas sembradas en el país (37 millones) ${ }^{49-50}$.

Tanto en 2009 como en 2015 -años de inicio de grandes epidemias de dengue en Argentina-, la principal provincia exportadora de semillas y frutos oleaginosos -entre los que se encuentra la soja- fue Santa Fe, seguida por Córdoba y Buenos Aires ${ }^{5 e}$, mientras que las zonas secundarias se ubican en las adyacencias de dichas provincias ${ }^{51-52}$.

Tanto Santa Fe, en 2009, como Córdoba, en 2015, representan aquellos casos provinciales donde la constatación de un antecedente de viaje de las personas enfermas nunca pudo ser una explicación suficiente para la incidencia y el aumento de confirmaciones. Esta conexión que surge directamente de los registros de los Partes de Situación y de los Boletines Integrados de Vigilancia apoya las observaciones y las denuncias de los estudios ${ }^{53-54}$ que señalan a la deforestación intensiva, que subyace a la instalación de modelos de agronegocio basados fundamentalmente en la soja, como un factor de creciente importancia en la expansión del dengue, tanto por la modificación del ecosistema

5c. En 2009, Santa Fe produjo el 54\% de los kilos netos de soja exportados, Córdoba el 22\% y, Buenos Aires el 14\% (en total, estas tres provincias concentraron el $90 \%$ del total del país). En 2015, Santa Fe produjo el 46\% de los kilos netos de soja exportados, Córdoba el 22\% y Buenos Aires el 21\% (en total, estas tres provincias concentraron el $89 \%$ del total del país). 
que ocasionó una mayor proliferación del mosquito vector, como por el cambio climático provocado por el uso de agrotóxicos y plaguicidas, que desencadena inundaciones y lluvias que no llegan a gestionarse con eficiencia y causan acumulaciones de agua en zonas con infraestructura deficiente.

\section{Conclusiones}

La información pública disponible permite reconstruir las vinculaciones entre epidemias y factores alternativos a las explicaciones hegemónicas, así como resaltan la predominancia insuficiente del argumento de la movilidad de personas en provincias donde estos casos no eran mayoritarios. Con relación a esto, la vinculación de focos epidémicos con la cercanía de la frontera se mantuvo como una segunda razón, pero varios brotes y focos de circulación del dengue tampoco pudieron explicarse mediante esas dos formulaciones.

La atención preponderante localizada sobre las trayectorias migratorias y la movilidad de personas impidió la construcción de explicaciones alternativas cuando los casos sin AV superaron ampliamente los casos con AV. En este punto se observan los obstáculos más fuertes y persistentes sobre la posibilidad de construir un relato explicativo sobre el dengue que incluya variables no incorporadas en la percepción hegemónica estatal, como es el caso de los argumentos científico-sociales que vinculan la prevalencia y la incidencia de dengue con los procesos estructurales de desigualdad socio-económica -que incide en las condiciones de infraestructura y vivienda-, y con el impacto ambiental producido por la profundización del modelo productivo de la soja en la región. Ambos argumentos permitirían complejizar la explicación diagnóstica sobre las epidemias de dengue, vinculando estos hallazgos con la formulación de políticas públicas integrales que observen la problemática en profundidad, atendiendo a sus heterogéneas y dinámicas dimensiones. De esta manera, sería posible elaborar una estrategia más compleja y amplia para gestionar y brindar soluciones tentativas a una problemática estructural de la salud pública en la región latinoamericana: la enfermedad del dengue.

\section{Bibliografía}

1. Sánchez Isaza DC, Chataño Ordoñez, N. Factores de riesgo total en familias migrantes en Soledad, Atlántico. Revista Cuidarte. 2016; 7(1): 1152-62.

2. Velasco-Mondragón HE, Johnson M, Chacón-Sosa F. Technology evaluation of a USA-Mexico health information system for epidemiological surveillance of Mexican migrant workers. Rev. Panam. Salud Pública. 2009; 7(3): 185-192.

3. Gomes Moreira J. Neoliberalismo y salud en la frontera de México y Estados Unidos. El caso de la tuberculosis. Migración y Desarrollo. Segundo semestre 2008; 55-78.

4. Suárez Larreinaga, CL, Berdasquera Corcho, D. Enfermedades emergentes y reemergentes: factores causales y vigilancia. Rev. Cubana Med. Gen. Integ. 2000; 16(6):593-597.

5. Sarmiento, MC. Enfermedades Transmisibles en Colombia: Cambios Ambivalentes. Salud Pública. 2000; 2(1): 82-93.

6. Estrada Márquez F, Magis Rodríguez C, Bravo García E. Epidemiología del VIH y migración. En Magis Rodríguez C y Barrientos Bárcenas H (Eds.) VIH-SIDA y salud pública: manual para el personal de salud. Cuernavaca: Instituto Nacional de Salud Pública; 2009.

7. Ministerio de Sanidad y Consumo. Enfermedades infecciosas importadas por viajeros internacionales a los trópicos. España; 2008. 
8. Ministerio de Sanidad y Consumo. Estudio de Inmigración y Salud Pública: enfermedades infecciosas importadas. España; 2007.

9. Organización Internacional para las Migraciones. Migración, salud y derechos humanos. Ginebra: OIM; 2013.

10. Canales A, Martínez Pizarro J, Reboiras Finardi L, Rivera Polo F. Migración y salud en zonas fronterizas: informe comparativo sobre cinco fronteras seleccionadas. Serie Población y Desarrollo. Santiago de Chile: CEPAL; 2010.

11. Jelin E (Dir.) Salud y migración regional: ciudadanía, discriminación y comunicación intercultural. Buenos Aires: IDES; 2006.

12. Demoscópica. Diagnóstico y factibilidad global para la implementación de políticas locales de salud para inmigrantes en la zona norte de la región metropolitana. Chile; OIM ChileMinisterio de Salud de Chile; 2009.

13. Breihl J. La determinación social de la salud como herramienta de transformación hacia una nueva salud pública (salud colectiva). Rev. Fac. Nac. Salud Pública. 2013; 31(supl 1): S13-S27.

14. Krieger N. Teorías para la epidemiología social en el siglo Xxi: una perspectiva ecosocial. International Journal of Epidemiology. Aug 2001; V30: 668-677.

15. Ortiz Z., Insúa I. Los meridianos de la pobreza y la enfermedad. El rol de la epidemiología en la medición de la vulnerabilidad social. Boletín de la Asociación Nacional de Medicina. 2003: 81(2); 239-255.

16. Vasilachis de Gialdino I. La investigación cualitativa. En Vasilachis de Gialdino I (Coord.) Estrategias de investigación cualitativa. Buenos Aires: Gedisa; 2007.
17. Mendizábal N. Los componentes del diseño flexible en la investigación cualitativa. En Vasilachis de Gialdino, I. (Coord.) Estrategias de investigación cualitativa. Buenos Aires: Gedisa; 2007.

18. Valles, M. Técnicas cualitativas de investigación social. Reflexión metodológica y práctica profesional. Madrid: Síntesis; 2014.

19. Charaudeau P., Maingueneau D. (Dirs.) Diccionario de Análisis del Discurso. Buenos Aires: Amorrortu Editores; 2002.

20. Maingueneau D. Términos clave de análisis del discurso. Buenos Aires: Nueva Visión; 2008.

21. Maingueneau D. ¿Situación de enunciación o situación de comunicación? Revista Discurso. 2004; 3(5) [citado el 10 octubre 2016]. Disponible en: http://es.slideshare.net/ MacueDeZan/98809453-maingueneaud2004 situaciondeenunciacionosituaciondecomunicacion.

22. Maingueneau D. Introducción a los métodos de análisis del discurso. Buenos Aires: Hachette; 1989.

23. Ducrot O. El decir y lo dicho. Buenos Aires: Edicial; 2001.

24. Arnoux E. Análisis del discurso. Modos de abordar materiales de archivo.Buenos Aires: Santiago Arcos Editor; 2009.

25. Matus C, Teoría del Juego Social. Lanús: Ediciones de la UNLa; 2007.

26. Ministerio de Salud de la Nación. Partes de Situación del Dengue en Argentina. Períodos seleccionados 2009-2013.

27. Ministerio de Salud de la Nación. Boletines de Vigilancia Epidemiológica. Periodos seleccionados 2015-2016. 
28. Abel L., Caggiano S. Enfermedades de Estado(s). Los inmigrantes y el acceso a la salud en una provincia de frontera. En Jelin E (Dir.) Salud y migración regional: ciudadanía, discriminación y comunicación intercultural. Buenos Aires: IDES; 2006.

29. Fantín MA. Disponibilidad y demanda de los servicios de salud en la frontera argentino-paraguaya. En II Taller "Paraguay como objeto de estudio de las Ciencias Sociales"; Asunción, mayo 2009. Grupo de Estudios Sociales sobre Paraguay; 2009.

30. Fantín MA, Meichtry N. Particularidades sociales y demográficas de la frontera argentino-paraguaya. En I Taller "Paraguay como objeto de estudio de las Ciencias Sociales", Resistencia, mayo. Grupo de Estudios Sociales sobre Paraguay; 2008.

31. Gottero L. Dengue en la agenda pública argentina: relatos institucionales sobre los brotes en la frontera noroeste (2009-2013). Punto Cero. 2015; año 20, $\mathrm{N}^{\circ}$ 31: 21-34.

32. Ortega Valcarcel J. Los horizontes de la geografía. En Ortega Valcárcel J Los horizontes de la geografía. Teoría de la geografía. Buenos Aires: Ariel; 2000.

33. Ramírez ML. La moderna geografía de la salud y las tecnologías de la información geográfica. Revista Investigaciones y Ensayos Geográficos. 2004; año IV, $\mathrm{N}^{\circ}$ 4: 53-64.

34. Iñiguez Rojas L. Geografía y salud: temas y perspectivas en América Latina. Cad. Saúde Publica. 1998: 14(4): 701-711.

35. De Almeida Filho N., Rouquayrol MZ. Perspectivas para una epidemiología de la salud. En De Almeida Filho N y Rouquayrol MZ Introducción a la epidemiología. Buenos Aires: Lugar Editorial; 2008.
36. Breihl J. Epidemiología crítica; ciencia emancipadora e interculturalidad. Buenos Aires: Lugar Editorial; 2009.

37. Timio M. Clases sociales y enfermedad. Introducción a una epidemiología diferencial. México: Editorial Nueva Imagen; 1979.

38. Menéndez E. De sujetos, saberes y estructuras. Buenos Aires: Lugar Editorial; 2009.

39. Terris M. La revolución epidemiológica y la medicina social. México: Siglo xxi Editores; 1987.

40. Buck C, Llopis A, Nájera E, Terris M. Servicios de salud y políticas de salud (discusión). En El desafío de la epidemiología. Problemas y lecturas seleccionadas. Washington DC: Organización Panamericana de la Salud; 1998.

41. Ortiz Z. Las enfermedades de la pobreza desde la Epidemiología. Revista Médicos Hoy. Marzo 2005 [citado 3 oct 2016] Disponible en: http://www.epidemiologia.anm.edu.ar/ pdf/publicaciones_cie/2005/enfermedades_ Pobreza_desde_Epidemiologia_2005.pdf.

42. Instituto Nacional de Estadísticas y Censos. Censo Nacional 2010, cuestionario básico, base de datos REDATAM [Internet] Buenos Aires: INDEC [citado 7 oct 2017].

43. Rosario Costa N. Os desafíos atuais da política pública para a dengue. Valor. 24/02/2011 [citado 5 oct 2016]; sección Económico. Disponible en: https://www.abrasco.org.br/ site/2016/02/desafios-atuais-da-politica-publica-para-a-dengue-por-nilson-do-rosario/

44. Neves Santos D. et. al. Documento de posición sobre la triple epidemia de Zika - Dengue - Chikungunya. Salvador: Observatorio de Análisis Político en Salud; 2015 [citado 25 sep 2016]. Disponible en https://www.abrasco.org.br/site/wp-content/uploads/2016/03/ 
Documento-posi\%C3\%A7\%C3\%A3osobre-a-epidemia-de-zika.pdf

45. Organización Mundial de Conservación. El crecimiento de la soja: impactos y soluciones. Suiza: WWF; 2014.

46. Dougnac Martínez G. Apuntes acerca de la historia de la soja en la Argentina. Elementos para delinear experiencias comparadas. Documentos del CIEA N $\mathrm{N}^{\circ}$ 2. Buenos Aires; 1994.

47. Cadenazzi G. La historia dela soja en Argentina. De los inicios al boom de los '90. XXVII Congreso de la Asociación Latinoamericana de Sociología. VIII Jornadas de Sociología de la Universidad de Buenos Aires. Asociación Latinoamericana de Sociología, Buenos Aires: 2009 [citado 29 sep 2016]. Disponible en: http://www.aacademica.org/000-062/394.pdf

48. Strada J, Vila IA. La producción de soja en Argentina: causas e impactos de su expansión. La revista del CCC. Julio / Diciembre 2015; 23 [citado 5 oct 2016]. Disponible en: http://www.centrocultural.coop/revista/ articulo/550/

49. Ybran R., Lacelli G. Informe estadístico mercado de la soja. Buenos Aires: INTA; 2016.
50. Centro deEconomía Política Argentina. La producción de soja en la Argentina. Buenos Aires: CEPA; 2014. Disponible en: http://centrocepa.com.ar/laproducciondesojaenlaargentina/

51. INDEC. Origen provincial de las exportaciones argentinas (OPEX). Sistema georreferenciado de consultas. Dirección URL: https:// opex.indec.gov.ar/index.php?pagina=mapa dinamico (última consulta: 15/9/2016).

52. INTAGRO. Atlas Agrícola [Internet]. 2015 [última consulta 8 sep 2017]. Disponible en: http://www.intagro.com/mapas/arg_soja.asp

53. Médicos del Mundo Argentina. Informe técnico sobre epidemia de dengue 2016. Buenos Aires: MDM [citado 15 sep 2016] Disponible en: http://www.mdm.org.ar/informes/37/ Documento-Tecnico-Evaluacion-Epidemiade-Dengue-20161.pdf

54. Avila Vazquez M. Informe de Médicos de Pueblos Fumigados sobre Dengue-Zika y fumigaciones con venenos químicos. REDUAS; 2016 [citado 7 oct 2016]. Disponible en: http://reduas.com.ar/informe-de-medicos-de-pueblos-fumigados-sobre-dengue-zika$\mathrm{y}$-fumigaciones-con-venenos-quimicos/ 